PROCEEDINGS OF THE

AMERICAN MATHEMATICAL SOCIETY

Volume 132, Number 6, Pages 1685-1691

S 0002-9939(04)07245-4

Article electronically published on January 16, 2004

\title{
SYMMETRY OF EXTREMAL FUNCTIONS FOR THE CAFFARELLI-KOHN-NIRENBERG INEQUALITIES
}

\author{
CHANG-SHOU LIN AND ZHI-QIANG WANG
}

(Communicated by David S. Tartakoff)

\begin{abstract}
We study the symmetry property of extremal functions to a family of weighted Sobolev inequalities due to Caffarelli-Kohn-Nirenberg. By using the moving plane method, we prove that all non-radial extremal functions are axially symmetric with respect to a line passing through the origin.
\end{abstract}

\section{INTRODUCTION}

This paper is concerned with symmetry properties of extremal functions for the following weighted Sobolev inequalities due to Caffarelli, Kohn and Nirenberg ([3]): for all $u \in C_{0}^{\infty}\left(\mathbb{R}^{N}\right)$,

$$
\left(\int_{\mathbb{R}}^{N}|x|^{-b p}|u|^{p} d x\right)^{2 / p} \leq C_{a, b} \int_{\mathbb{R}}^{N}|x|^{-2 a}|\nabla u|^{2} d x
$$

where for $N \geq 3$ :

$$
-\infty<a<\frac{N-2}{2}, \quad a \leq b \leq a+1, \quad \text { and } p=\frac{2 N}{N-2+2(b-a)},
$$

and for $N=2$ :

$$
-\infty<a<0, \quad a<b \leq a+1, \quad \text { and } p=\frac{2}{b-a} .
$$

Let $\mathcal{D}_{a}^{1,2}\left(\mathbb{R}^{N}\right)$ be the completion of $C_{0}^{\infty}\left(\mathbb{R}^{N}\right)$, with respect to the inner product

$$
(u, v)_{a}=\int_{\mathbb{R}}^{N}|x|^{-2 a} \nabla u \cdot \nabla v d x .
$$

Then inequalities (11) are extended to all $u \in \mathcal{D}_{a}^{1,2}\left(\mathbb{R}^{N}\right)$. Define

$$
S(a, b)=\inf _{u \in \mathcal{D}_{a}^{1,2}\left(\mathbb{R}^{N}\right) \backslash\{0\}} E_{a, b}(u)
$$

Received by the editors October 30, 2002.

2000 Mathematics Subject Classification. Primary 35B33; Secondary 46 E35.

Key words and phrases. Weighted Sobolev inequalities, Extremal functions, Exact symmetry. 
to be the best embedding constants, where

$$
E_{a, b}(u)=\frac{\int_{\mathbb{R}}^{N}|x|^{-2 a}|\nabla u|^{2} d x}{\left(\int_{\mathbb{R}}^{N}|x|^{-b p}|u|^{p} d x\right)^{2 / p}} .
$$

The extremal functions for $S(a, b)$ are least-energy solutions of the Euler equation

$$
-\operatorname{div}\left(|x|^{-2 a} \nabla u\right)=|x|^{-b p} u^{p-1}, \quad u \geq 0, \quad \text { in } \mathbb{R}^{N} .
$$

The best constant and the minimizers for the Sobolev inequality $(a=b=0)$ were given by Aubin [1] and Talenti [16]. In [13, Lieb considered the case $a=0$, $0<b<1$ and gave the best constants and explicit minimizers. In [8, Chou and Chu considered the $a$-nonnegative region and gave the best constants and explicit minimizers. The symmetry of the minimizers has also been studied in [13] and [8]. In summary, for $a \geq 0$, all nonnegative solutions in $\mathcal{D}_{a}^{1,2}\left(\mathbb{R}^{N}\right)$ for the corresponding Euler equation (7) are radial solutions (in the case $a=b=0$, they are radial with respect to some point) and explicitly given ([1], [16, [13, [8]). This was established in [8], using a generalization of the moving plane method (e.g., [10, 11], 2]).

Recently, Catrina and Wang (4, 5]) have discovered the symmetry-breaking phenomenon of the extremal functions for a sub-region of parameters when $a<0$ occurs (see also [19] for a partial result). More precisely, they proved in [4], [5] that there is a function $h(a)$ defined for $a \leq 0$, satisfying $h(0)=0, a<h(a)<a+1$ for $a<0$, and $a+1-h(a) \rightarrow 0$ as $-a \rightarrow \infty$, such that for any $(a, b)$ satisfying $a<0$ and $a<b<h(a)$, the extremal function for $S(a, b)$ is non-radial. In a recent preprint [9] of Felli and Schneider, it is observed that this curve $h(a)$ can be sharpened to the following:

$$
h(a)=1+a-\frac{N}{2}\left(1-\frac{N-2-2 a}{\sqrt{(N-2-2 a)^{2}+4(N-1)}}\right) .
$$

A natural question is on the symmetry of these non-radial extremal functions. In 6], Catrina and Wang have proved that for $c \in(0,1)$ fixed, for sufficiently large $-a$, up to rotations and dilations, the extremal function to $S(a, a+c)$ is unique and has $\mathcal{O}(N-1)$ symmetry (i.e., the extremal function is axially symmetric with respect to a line passing through the origin).

In this paper, the main result is the following that gives the exact symmetry for all non-radial extremal functions.

Theorem 1.1. Let $N \geq 2$. For all $(a, b)$ satisfying $a<0$ and $a<b<h(a)$, the extremal function $u$ to $S(a, b)$ has exact $\mathcal{O}(N-1)$ symmetry. More precisely, up to a rotation, $u(x)$ only depends on the radius $r$ and the angle $\theta_{N}$ between the positive $x_{N}$-axis and $\overrightarrow{O x}$, and on each sphere $\left\{x \in \mathbb{R}^{N}|| x \mid=r\right\}, u$ is strictly decreasing as the angle $\theta_{N}$ increases, i.e., $u=u\left(\theta_{N}, r\right)$ and $\frac{\partial u}{\partial \theta_{N}}\left(\theta_{N}, r\right)<0$ for all $r>0$ and all $\theta_{N} \in(0, \pi)$.

Another natural question is what is the symmetry of the extremal functions for the parameters $(a, b)$ with $h(a) \leq b<a+1$ for $a<0$. The following result offers a partial answer. 
Theorem 1.2. Let $N \geq 3$, and $b_{0} \in(0,1)$ be fixed. Then with $C>0$ given, for all $(a, b)$ sufficiently close to $\left(0, b_{0}\right)$, any bound state solution of equation $u$ of (7) satisfying $\|u\|_{a} \leq C$ is radially symmetric with respect to the origin.

This theorem improves the result of [17] in which they obtained a result for the least-energy solutions. Our argument is different from that in [17].

In Section 2 we first recall a new formulation of the inequalities (1) from [4], [5]. For this alternative formulation some new ideas developed in [7], [15] for proving symmetry properties via the maximum principle can be applied to derive the desired results. Then we shall give the proofs of the main theorems.

\section{Proofs of the MAin Results}

We start by recalling a reformulation of the inequalities (1) given in [4, [5]. The proofs of our main results will be based on this new formulation.

We shall use the notation $x=(\theta, t) \in \mathcal{C}=\mathbf{S}^{N-1} \times \mathbb{R}$, which is placed in $\mathbb{R}^{N+1}$ with the $t$-axis coinciding with the $x_{N+1}$-axis in $\mathbb{R}^{N+1}$. To $u$ a smooth function with compact support in $\mathbb{R}^{N} \backslash\{0\}$, we associate $v$ a smooth function with compact support on $\mathcal{C}$, by the transformation

$$
u(y)=|y|^{-\frac{N-2-2 a}{2}} v\left(\frac{y}{|y|},-\ln |y|\right) .
$$

Here for $y \in \mathbb{R}^{N} \backslash\{0\}$, with $t=-\ln |y|$ and $\theta=\frac{y}{|y|}$ we have $(\theta, t) \in \mathcal{C}$. It was proved that the mapping (9) is a Hilbert space isomorphism from $\mathcal{D}_{a}^{1,2}\left(\mathbb{R}^{N}\right)$ to $H^{1}(\mathcal{C})$, where the inner product on $H^{1}(\mathcal{C})$ is

$$
(v, w)=\int_{\mathcal{C}} \nabla v \cdot \nabla w+\left(\frac{N-2-2 a}{2}\right)^{2} v w d \mu .
$$

If there is no ambiguity, we still use $\|\cdot\|_{a}$ for the norm in $H^{1}(\mathcal{C})$ with respect to the above inner product.

Now we define an energy functional on $H^{1}(\mathcal{C})$ by

$$
F_{a, b}(v)=\frac{\int_{\mathcal{C}}\left|\nabla_{\theta} v\right|^{2}+v_{t}^{2}+\left(\frac{N-2-2 a}{2}\right)^{2} v^{2} d \mu}{\left(\int_{\mathcal{C}} v^{p} d \mu\right)^{2 / p}} .
$$

If $u \in \mathcal{D}_{a}^{1,2}\left(\mathbb{R}^{N}\right)$ and $v \in H^{1}(\mathcal{C})$ are related through (9), then

$$
E_{a, b}(u)=F_{a, b}(v) .
$$

Moreover, if $u$ is a solution of (7), then $v$ satisfies

$$
-v_{t t}-\Delta_{\theta} v+\left(\frac{N-2-2 a}{2}\right)^{2} v=v^{p-1}, v>0, \quad \text { on } \mathcal{C}
$$

where $t=-\ln |y|$, and $\Delta_{\theta}$ is the Laplace operator on the $N-1$ sphere. In summary we have the following from [4], [5].

Proposition 2.1. (i) The transformation (91) gives a Hilbert space isomorphism between $\mathcal{D}_{a}^{1,2}\left(\mathbb{R}^{N}\right)$ and $H^{1}(\mathcal{C})$.

(ii) If $u \in \mathcal{D}_{a}^{1,2}\left(\mathbb{R}^{N}\right)$ and $v \in H^{1}(\mathcal{C})$ are related through (9), then $E_{a, b}(u)$ $=F_{a, b}(v)$. Therefore, for $S(a, b)$ as defined in (5), it follows that $S(a, b)=$ $\inf _{H^{1}(\mathcal{C}) \backslash\{0\}} F_{a, b}(v)$. 
(iii) Solutions of (7) and (11) are in one-to-one correspondence, being related through (9).

We shall prove the following result regarding the new formulation, which implies Theorem [1.1 By the result in [5], without loss of generality, we may assume that any solution of (11) achieves its maximum at $t=0$, and is even in $t$ and monotonic decreasing for $t>0$. For $t \in \mathbb{R}$ we denote by $O_{t}$ the point on the $x_{N+1}$-axis in $\mathbb{R}^{N+1}$ with coordinate $t$, i.e., $O_{t}=(0, \ldots, 0, t)$.

Theorem 2.2. Let $N \geq 2$. Let $v$ be a least-energy solution of (11) such that $v$ is non-radial (i.e., $v$ depends on $\theta$ ), and let $P_{0}$ be a maximum point of $v$. Then for each fixed $t, v(\theta, t)$ is axially symmetric with respect to the line passing through $O_{t}$ and $P_{0}+O_{t}$. Moreover, by assuming that $P_{0}$ is located on the positive $x_{N}$ axis and denoting by $\theta_{N}$ the angle between the vector from $O_{t}$ to $P_{0}+O_{t}$ and the vector from $O_{t}$ to $x$ with $x=(\theta, t)$, we have that $v$ depends only on $t$ and $\theta_{N}$, and $\frac{\partial v}{\partial \theta_{N}}\left(\theta_{N}, t\right)<0$ for all $\theta_{N} \in(0, \pi)$ and all $t \in \mathbb{R}$.

We shall follow the ideas developed recently in [7, 15], which are along the line of research of using the moving plane method for symmetry of positive solutions (10], [1] $)$.

Proof. For simplicity, we write $\lambda_{a}=\left(\frac{N-2-2 a}{2}\right)^{2}$. Let $T$ be any hyperplane in $\mathbb{R}^{N+1}$ that passes through the $x_{N+1}$-axis. Let $\mathcal{C}^{+}$be one of the half cylinders of $\mathcal{C} \backslash T$. For $x \in \mathcal{C}^{+}, x^{*}$ denotes the reflection point of $x$ with respect to the plane $T$.

We claim that one of the following assertions holds: (a) $v(x)=v\left(x^{*}\right)$ for all $x \in \mathcal{C}^{+} ;$(b) $v(x)>v\left(x^{*}\right)$ for all $x \in \mathcal{C}^{+} ;(\mathrm{c}) v(x)<v\left(x^{*}\right)$ for all $x \in \mathcal{C}^{+}$.

To this end, we first prove that either $v(x) \geq v\left(x^{*}\right)$ for all $x \in \mathcal{C}^{+}$, or $v(x) \leq v\left(x^{*}\right)$ for all $x \in \mathcal{C}^{+}$. Suppose this is not true. Then the following two sets are both nonempty:

$$
D_{+}=\left\{x \in \mathcal{C}^{+} \mid v(x)>v\left(x^{*}\right)\right\}, \quad D_{-}=\left\{x \in \mathcal{C}^{+} \mid v(x)<v\left(x^{*}\right)\right\} .
$$

Define $w(x)=v(x)-v\left(x^{*}\right)$ for $x \in \mathcal{C}^{+}$. Then $w$ satisfies

$$
\begin{cases}-\Delta w+\lambda_{a} w=c(x) w, & x \in \mathcal{C}^{+}, \\ w=0, & x \in \partial \mathcal{C}^{+}\end{cases}
$$

where $c(x)=(p-1) \int_{0}^{1}\left(s v(x)+(1-s) v\left(x^{*}\right)\right)^{p-2} d s$. Denote $D_{-}^{*}=\left\{x^{*} \mid x \in D_{-}\right\}$ and define $u$ on $\mathcal{C}$ as follows:

$$
u(x)= \begin{cases}w(x), & x \in D_{+}, \\ d w\left(x^{*}\right), & x \in D_{-}^{*}, \\ 0, & \text { otherwise. }\end{cases}
$$

Here the constant $d>0$ is chosen such that

$$
\int_{\mathcal{C}} u(x) \phi_{1}(x)=0
$$

with $\phi_{1}$ being the first eigenfunction of the following eigenvalue problem:

$$
\begin{cases}-\Delta \phi+\lambda_{a} \phi-(p-1) v^{p-2} \phi=\mu \phi, & x \in \mathcal{C} \\ \phi=0, & x \in \partial \mathcal{C}\end{cases}
$$


which is well-defined due to the decay property of $v$ as $|t| \rightarrow \infty$. Let $\mu_{2}$ be the second eigenvalue of (14). Since $v$ is a least-energy solution of (11) we have $\mu_{2} \geq 0$. On the other hand, we may check that $u$ is not identically zero and

$$
-\Delta u+\lambda_{a} u-(p-1) v^{p-2} u \begin{cases}\leq 0, & x \in D_{+}, \\ \geq 0, & x \in D_{-}^{*}, \\ =0, & \text { otherwise. }\end{cases}
$$

Following these we have a contradiction as follows:

$0>\int_{\mathcal{C}} u\left(-\Delta u+\lambda_{a} u-(p-1) v^{p-2} u\right) d x=\int_{\mathcal{C}}\left(|\nabla u|^{2}+\lambda_{a} u^{2}-(p-1) v^{p-2} u^{2}\right) d x \geq 0$.

Thus we have proved that either $w \geq 0$ or $w \leq 0$ for $x \in \mathcal{C}^{+}$. By the strong maximum principle, we have either $w>0$, or $w<0$, or $w=0$ for all $x \in \mathcal{C}^{+}$, corresponding to the three alternatives in the claim. This proves the claim.

Next, let $P_{0}$ be a maximum point of $v$. Without loss of generality, we may assume that $P_{0}=(0, \ldots, 0,1,0) \in \mathbb{R}^{N+1}$, i.e., $P_{0}$ is on the $x_{N}$-axis. Let $T_{0}$ be the hyperplane in $\mathbb{R}^{N+1}$ that has $x_{N}$ as its normal direction. Let $\mathcal{C}^{+}=\left\{x \in \mathcal{C} \mid x_{N}>0\right\}$ and for $x \in \mathcal{C}^{+}$, let $x^{-}$be the reflection point of $x$ with respect to $T_{0}$. Then from the first part of the proof we have $v(x)>v\left(x^{-}\right)$for all $x \in \mathcal{C}^{+}$. Since otherwise, $v\left(P_{0}\right)=v\left(P_{0}^{-}\right)=\max _{\mathcal{C}} v$ and we may produce a contradiction as follows. Let $T$ be any hyperplane in $\mathbb{R}^{N+1}$ that contains the $x_{N+1}$-axis such that $P_{0} \notin T$, and let $\mathcal{C}_{T}$ be the half cylinder of $\mathcal{C} \backslash T$ such that $P_{0} \in \mathcal{C}_{T}$. For $x \in \mathcal{C}_{T}$, let $x^{*}$ denote the reflection point of $x$ with respect to $T$. Then we have $v\left(P_{0}\right) \geq v\left(P_{0}^{*}\right)$ and $v\left(\left(P_{0}^{-}\right)^{*}\right) \leq v\left(P_{0}^{-}\right)$. By the proof in the first part, we obtain $v(x)=v\left(x^{*}\right)$ for all $x \in \mathcal{C}_{T}$. Since $T$ is arbitrary, we assert that $v$ must be independent of its $\theta$ component, which is a contradiction with the assumption.

Finally, choose any two-dimensional plane in $\mathbb{R}^{N}$ that contains the $x_{N}$-axis. For simplicity we assume this is generated by the $x_{1}$ and $x_{N}$ directions. Let $R_{\omega}$ be the ray from the origin on this two-dimensional plane that has an angle $\omega$ with the positive $x_{1}$ direction for $\omega \in\left(-\frac{\pi}{2}, \frac{\pi}{2}\right)$ with $\omega=\frac{\pi}{2}$ corresponding to the positive $x_{N}$-axis. Let $\nu_{\omega}$ be the normal vector of the ray $R_{\omega}$ in this two-dimensional plane with $\nu_{0}=e_{N}$ (the $N$-th axis direction) and let $T_{\omega}$ be the $N$-dimensional hyperplane in $\mathbb{R}^{N+1}$ that has $\nu_{\omega}$ as its normal vector. Let $\mathcal{C}_{\omega}$ be the half cylinder of $\mathcal{C} \backslash T_{\omega}$ that contains $P_{0}$ for $\omega \in\left[0, \frac{\pi}{2}\right)$, and for $x \in \mathcal{C}_{\omega}$ let $x^{\omega}$ be the reflection point of $x$ with respect to $T_{\omega}$. Define $u_{\omega}(x)=v(x)-v\left(x^{\omega}\right)$ for $x \in \mathcal{C}_{\omega}$. Then we have

$$
\begin{cases}-\Delta u_{\omega}+\lambda_{a} u_{\omega}-c_{\omega}(x) u_{\omega}=0, & x \in \mathcal{C}_{\omega} \\ u_{\omega}=0, & x \in \partial \mathcal{C}_{\omega}\end{cases}
$$

where $c_{\omega}(x)=(p-1) \int_{0}^{1}\left(s v(x)+(1-s) v\left(x^{\omega}\right)\right)^{p-2} d s$. For $\omega=0$, we have $u_{0}(x)>0$ for all $x \in \mathcal{C}_{0}$. Let

$$
\omega_{0}=\sup \left\{\omega \mid u_{\omega^{\prime}}(x) \geq 0, \forall x \in \mathcal{C}_{\omega^{\prime}}, \forall 0 \leq \omega^{\prime} \leq \omega \leq \frac{\pi}{2}\right\} .
$$

We want to prove $\omega_{0}=\frac{\pi}{2}$. If this is not true, we produce a contradiction as follows. From the definition of $\omega_{0}$ and the proofs in the first part we have for $0 \leq \omega<\omega_{0}$, $u_{\omega}(x)>0$ for $x \in \mathcal{C}_{\omega}, \frac{\partial u}{\partial \nu_{\omega}}(x)>0$ for $x \in T_{\omega}$, and $u_{\omega_{0}}(x)=0$ for all $x \in \mathcal{C}_{\omega_{0}}$. Since $P_{0}^{\omega_{0}}$ is also a global maximum point of $v$, we have $P_{0}^{\omega_{0}} \in T_{\omega_{1}}$ for some $\omega_{1} \in\left(0, \omega_{0}\right)$ and $\nabla v\left(P_{0}^{\omega_{0}}\right)=0$. We have a contradiction with $2 \frac{\partial v}{\partial \nu_{\omega_{1}}}\left(P_{0}^{\omega_{0}}\right)=\frac{\partial u}{\partial \nu_{\omega_{1}}}\left(P_{0}^{\omega_{0}}\right)>0$. 
Therefore we obtain $u_{\frac{\pi}{2}}(x) \geq 0$ in $\mathcal{C}_{\frac{\pi}{2}}$. Using the same argument we may obtain also $u_{-\frac{\pi}{2}}(x) \geq 0$ in $\mathcal{C}_{-\frac{\pi}{2}}$. This leads to $u_{\frac{\pi}{2}}(x)=0$ for all $x \in \mathcal{C}_{\frac{\pi}{2}}$. Now it follows that for all $\omega \in\left(-\frac{\pi}{2}, \frac{\pi}{2}\right), 2 \frac{\partial v}{\partial \omega}(x)=\frac{\partial u}{\partial \nu_{\omega}}(x)>0$ for all $x \in T_{\omega}$. Note that for $t$ fixed, the angle $\theta_{N}$ between the vector from $O_{t}$ to $P_{0}+O_{t}$ and the vector from $O_{t}$ to the point $x$ is given by $\theta_{N}=\frac{\pi}{2}-\omega$; so we get $\frac{\partial v}{\partial \theta_{N}}(x)<0$. This completes the proof of Theorem 2.2.

Remark. The method above was used in 7. for more general nonlinearity $f(v)$ than $v^{p-1}$. This also applies to our equation (11). In fact, the same result of Theorem 2.2 still holds for equation (11) under the following conditions on $f(v): f \in$ $C^{2}(\mathbb{R}, \mathbb{R}), f^{\prime}(0)=0$, there is $\theta \in\left(0, \frac{1}{2}\right)$ such that $0<F(u)=\int_{0} u f(s) d s \leq \theta u f(u)$ for all $u \neq 0$, and there are $C>0$ and $q \in\left(2,2^{*}\right)$ such that $|f(u)| \leq C\left(1+|u|^{q-1}\right)$. We leave the details of the statements to the reader. We do not know whether such a result is still true for equation (77).

Finally we give the proof of Theorem 1.2 .

Proof. Again we shall work with equation (11). First we observe that for $\frac{N-2}{2}>$ $a \geq 0, a \leq b<a+1$ and $a+b \neq 0$, when we make a linearization at the radial solution of equation (11), the kernel is generated only by the translation invariance in $t$ of the problem (see [17], [18). Thus if we confine ourselves to the subspace of even functions in $t$, the radial solution is nondegenerate in the sense that the kernel of the linearization is trivial, and therefore the Implicit Functions Theorem can be applied at these parameters. Since there is a unique radial solution (in the even functions space) we conclude that the radial solutions are isolated. By the result of [5] that any (even) solution is monotonically decreasing to zero as $|t| \rightarrow \infty$, we have that for $(a, b)$ near $\left(0, b_{0}\right)$, all solutions uniformly tend to zero as $|t| \rightarrow \infty$. Let $C>0$ be fixed. Now if for a sequence $\left(a_{n}, b_{n}\right) \rightarrow\left(0, b_{0}\right)$ with $a_{n}<0$ (in equation (11) this corresponds to $p_{n} \rightarrow p_{0}=\frac{2 N}{N-2+2 b_{0}} \in\left(2,2^{*}\right)$ ), there are non-radial solutions $v_{n}$ for these parameters satisfying $\left.\left\|v_{n}\right\|\right|_{a_{n}} \leq C$, then we must have for a subsequence, $\max _{\mathcal{C}} v_{n}=M_{n} \rightarrow \infty$. Otherwise, due to the energy bound and using the concentration-compactness arguments we would get a sequence of non-radial solutions converging to the even (nontrivial) radial solution at $\left(0, b_{0}\right)$, which is a contradiction with the fact that even radial solutions are isolated. Without loss of generality, we assume that a maximum point of $v_{n}$ is at $P=(1,0, \ldots, 0) \in \mathcal{C} \subset \mathbb{R}^{N+1}$ and for given $0<r<1$ we define a map from $B_{r}(P):=\left\{x \in \mathcal{C} \mid\left(x_{1}-1\right)^{2}+x_{2}^{2}+\ldots+x_{N+1}^{2}<r^{2}\right\}$ onto $B_{r}(0) \subset \mathbb{R}^{N}$ by $\psi(x)=\left(x_{2}, \ldots x_{N}, x_{N+1}\right) \in \mathbb{R}^{N}$. It is easy to see that $\psi$ has an inverse map and the Jacobians of both $\psi$ and $\psi^{-1}$ are 1 at $P$ and 0 respectively. Define

$$
w(x)=M_{n}^{-1} v_{n}\left(\psi^{-1}\left(M_{n}^{-\frac{p_{n}-2}{2}} x\right)\right), \text { for } x \in \mathbb{R}^{N} \text {, with }|x|<M_{n}^{\frac{p_{n}-2}{2}} r .
$$

Then $0 \leq w_{n} \leq 1$ and $w_{n}(0)=1$ and $w_{n}$ satisfies an elliptic equation of the form

$$
-\sum_{i, j=2}^{N+1} a_{i, j, n}(x) \frac{\partial w_{n}}{\partial x_{i} \partial x_{j}}+\sum_{i=2}^{N+1} b_{i, n}(x) \frac{\partial w_{n}}{\partial x_{i}}+c_{n} w_{n}=w_{n}^{p_{n}-1} .
$$

Using the fact that the Jacobians of $\psi$ and $\psi^{-1}$ both tend to 1 at $P$ and 0 respectively, we have $a_{i, j, n}(x) \rightarrow I d_{N \times N}, b_{i, n}(x) \rightarrow 0, c_{n}(x) \rightarrow 0$, as $n \rightarrow \infty$, all uniformly in a bounded set of $x$. By elliptic theory we have that $w_{n}$ converges to $w$ in $C_{l o c}^{2}\left(\mathbb{R}^{N}\right)$ and $w$ is a positive solution of $-\Delta w=w^{p_{0}-1}$ in $\mathbb{R}^{N}$. However, 
this is a contradiction since it is well known that such a solution does not exist for $p_{0} \in\left(2,2^{*}\right)$. This completes the proof of Theorem [1.2.

The question on the symmetry of extremal functions for $h(a) \leq b<a+1$, in general, remains open.

\section{ACKNOWLEDGMENT}

The second author would like to thank the kind hospitality of the National Center for Theoretical Sciences, Taiwan, where this work was initiated during his visit.

\section{REFERENCES}

[1] T. Aubin, Problèmes isopérimétriques et espaces de Sobolev, J. Differential Geometry, 11 (1976), 573-598. MR 56:6711

[2] L. A. Caffarelli, B. Gidas, and J. Spruck, Asymptotic symmetry and local behavior of semilinear elliptic equations with critical Sobolev growth, Comm. Pure Appl. Math., 42 (1989), 271-297. MR 90c:35075

[3] L. Caffarelli, R. Kohn, and L. Nirenberg, First order interpolation inequalities with weights, Compositio Mathematica, 53 (1984), 259-275. MR 86c:46028

[4] F. Catrina and Z.-Q. Wang, On the Caffarelli-Kohn-Nirenberg Inequalities, Comptes Rendus des Séances de l'Académie des Sciences. Sér. I. Math., 330 (2000), 437-442. MR 2001h:46047

[5] F. Catrina and Z.-Q. Wang, On the Caffarelli-Kohn-Nirenberg inequalities: sharp constants, existence (and nonexistence), and symmetry of extremal functions, Comm. Pure Appl. Math., 54 (2001), 229-258. MR 2001k:35028

[6] F. Catrina and Z.-Q. Wang, Asymptotic Uniqueness and Exact Symmetry of k-bump Solutions for a Class of Degenerate Elliptic Problems, Discrete Contin. Dynam. Systems, Added Vol. (2001), 80-87.

[7] J.-L. Chern and C.-S. Lin, The symmetry of least-energy solutions for semilinear elliptic equations, J. Differential Equations, 187 (2003), 240-268. MR 2003m:35065

[8] K. S. Chou and C. W. Chu, On the best constant for a weighted Sobolev-Hardy inequality, J. London Math. Soc. (2) 48 (1993), 137-151. MR 94h:46052

[9] V. Felli and M. Schneider, Perturbation results of critical elliptic equations of CaffarelliKohn-Nirenberg type, J. Differential Equations, 191 (2003), 121-142.

[10] B. Gidas, W.-M. Ni, and L. Nirenberg, Symmetry and related properties via the maximum principle, Comm. Math. Phys., 68 (1979), 209-243. MR 80h:35043

[11] B. Gidas, W.-M. Ni, and L. Nirenberg, Symmetry of positive solutions of nonlinear elliptic equations in $\mathbb{R}^{n}$, Adv. in Math. Suppl. Studies, 7A (1981), 369-402. MR 84a:35083

[12] T. Horiuchi, Best constant in weighted Sobolev inequality with weights being powers of distance from the origin, J. Inequal. and Appl., 1 (1997), 275-292. MR 2000k:35110

[13] E. H. Lieb, Sharp constants in the Hardy-Littlewood-Sobolev and related inequalities, Annals of Math., 118 (1983), 349-374. MR 86i:42010

[14] C. S. Lin, Interpolation inequalities with weights, Comm. Partial Differential Equations, 11 (1986), 1515-1538. MR 88a:46037

[15] C. S. Lin, Locating the peaks of solutions via the maximum principle: I. The Neumann problem, Comm. Pure Appl. Math., 54 (2001), 1065-1095. MR 2002d:35052

[16] G. Talenti, Best constant in Sobolev inequality, Ann. Mat. Pura Appl., 110 (1976), 353-372. MR 57:3846

[17] D. Smets and M. Willem, Partial symmetry and asymptotic behavior for some elliptic variational problems, Calc. Var. Partial Differential Equations 18 (2003), 57-75.

[18] Z.-Q. Wang and M. Willem, Caffarelli-Kohn-Nirenberg inequalities with remainder terms, J. Funct. Anal. 203 (2003), 550-568.

[19] M. Willem, A decomposition lemma and critical minimization problems, preprint.

Department of Mathematics, National Chung Cheng University, Chiayi, Taiwan

Department of Mathematics and Statistics, Utah State University, Logan, Utah 84322 